DNA AND CELL BIOLOGY

Volume 27, Number 11, 2008

(c) Mary Ann Liebert, Inc.

Pp. 607-614

DOI: $10.1089 /$ dna.2008.0773
Scott, D.W., Longpre, J.M., Loo, G. (2008) Upregulation of GADD153 by butyrate: involvement of MAPK. DNA and Cell Biol. 27 , 607-614. DOI: $10.1089 /$ dna.2008.0773

Made available courtesy of Mary Ann Liebert, Inc.: http://www.liebertpub.com/

\title{
Upregulation of GADD153 by Butyrate: Involvement of MAPK
}

\author{
David W. Scott, Jennifer M. Longpre, and George Loo
}

Butyrate inhibits the proliferation of cancer cells, but the early molecular events initiated by butyrate have not been fully identified. Herein, butyrate is shown to affect the growth arrest and DNA damage-inducible gene 153 (GADD153) in HCT-116 human colon adenocarcinoma cells. Despite absence of any detectable cellular DNA damage, the expression of GADD153 was upregulated before several features characteristic of apoptosis appeared. Butyrate-induced upregulation of GADD153 mRNA was attenuated by actinomycin D, but apparently not by cycloheximide. In investigating possible involvement of MAPK in mediating the effect of butyrate on GADD153 mRNA expression, the extracellular regulated kinase (ERK) inhibitor PD98059, but neither the JNK inhibitor SP600125 nor the p38 MAPK inhibitor SB203580, blunted the ability of butyrate to upregulate GADD153 mRNA expression. U0126, a selective inhibitor of upstream MEK, had a similar effect as PD98059 on butyrate-induced GADD153 mRNA upregulation. Collectively, these findings suggest that butyrate caused activation of the GADD153 gene at the level of transcription involving mainly the MEK/ERK branch of the MAPK signal transduction pathway. Moreover, these molecular events were not the result of any DNA damage and occurred before several features characteristic of apoptosis became evident.

\section{Introduction}

$\mathbf{T}$ He FOUR-CARBON SATURATED carboxylic acid, butyrate, is often recognized as a compound that can suppress the growth or proliferation of cancer cells by different means. Butyrate affects the expression of genes that regulate the cell cycle. For example, butyrate inhibited the proliferation of human colon adenocarcinoma cells by inducing p21 (WAF1) gene expression (Nakano et al., 1997; Archer et al., 1998). Moreover, this effect of butyrate was similar to trichostatin A, a specific histone deacetylase inhibitor, suggesting that butyrate promoted histone hyperacetylation in producing its molecular effect. Apparently, butyrate creates a cellular environment where the cis-elements localized in the promoter region of the $p 21$ gene become more accessible for binding to the appropriate transcription factors that are involved in $p 21$ gene activation. Whereas p53 is not critical (Nakano et al., 1997; Archer et al., 1998), at least three other transcription factors have been considered as potential mediators of butyrate-induced $p 21$ gene activation. Sp1 seems to be involved, based on the original finding of at least two Sp1 sites in the $p 21$ gene promoter (Nakano et al., 1997; Sowa et al., 1997) that constitute butyrate-responsive cis-elements. On the other hand, it has been reported that Sp3, rather than Sp1, could be more pivotal in $p 21$ gene activation by butyrate (Sowa et al.,
1999). A role for ZBP-89 has also been reported (Bai and Merchant, 2000). Following transcriptional activation and translation of transcript, p21 protein triggers cell cycle arrest at the $G_{1}$ phase by inhibiting cyclin-dependent kinases.

In considering other effects of butyrate in inhibiting cancer cell proliferation, it is known that butyrate can promote differentiation in certain cancer cell lines, such as Caco-2 colonocytes (Litvak et al., 1998). Alternatively, butyrate can induce apoptosis in Caco-2 colonocytes, which was reported to occur via the mitochondrial pathway involving release of cytochrome c and activation of caspases (Ruemmele et al., 2003). The molecular mechanism of the apoptotic effect of butyrate is unclear, but it has been reported that butyrateinduced apoptosis happens in association with downregulated expression of the antiapoptotic genes, $\mathrm{Bcl}-2$ and Bcl-XL (Litvak et al., 1998). In analyzing the expression of gene products, butyrate has been shown to simultaneously increase expression of the proapoptotic protein, bak, and decrease the expression of bcl-xl protein in inducing caspasemediated apoptosis (Ruemmele et al., 2003).

It is likely that butyrate stimulates early-response events that precede the initiation of cell cycle arrest and/or cell death. To gain a better understanding along this line, the primary aim of the present study was to determine if butyrate influences the expression of a specific member of the growth 
arrest and DNA damage-inducible genes (GADD). GADD expression is often upregulated a relatively short time after cells have been subjected to a stressful or potentially lethal environment, such as created by a strong oxidant (Oh-Hashi et al., 2001) or genotoxic compound (Scott et al., 2005). DNA microarray analysis revealed that butyrate upregulates the GADD45A gene in HCT-116 human colon adenocarcinoma cells (Daly and Shirazi-Beechey, 2006). We tested the hypothesis that exposing HCT-116 cells to butyrate would upregulate the GADD153 gene with an involvement of a mitogen-activated protein kinase (MAPK). It was found that butyrate stimulates extracellular regulated kinase (ERK)dependent induction of GADD153 mRNA expression, as part of an apparent molecular cascade initiated not as a result of any DNA damage and happening before the subsequent onset of apoptosis.

\section{Materials and Methods}

\section{Materials}

The HCT-116 human colon adenocarcinoma cell line was obtained from the American Type Culture Collection (Manassas, VA). PD98059 and SB203580 were purchased from Calbiochem (San Diego, CA), and SP600125 from Tocris (Ellisville, MO). U0126 was purchased from BioMol (Plymouth Meeting, PA). Butyrate and all other reagents were purchased from Sigma Chemical (St. Louis, MO) unless otherwise stated.

\section{Cell culture and treatment}

HCT-116 colonocytes were propagated in McCoy's 5A medium (Sigma) that was supplemented with $100 \mathrm{M}$ fetal bovine serum (BioWhittaker, Walkersville, MD), $2 \mathrm{mM}$ glutamine, $0.54 \mathrm{M}$ fungizone, 100,000 units/L penicillin, and $100 \mathrm{mg} / \mathrm{L}$ streptomycin (last four items from Atlanta Biologicals, Atlanta, GA). Upon reaching $70-80 \%$ confluency, the cells were exposed to $0-16 \mathrm{mM}$ butyrate for $0-48 \mathrm{~h}$ depending on the experiment. Cells were also exposed to $10 \mu \mathrm{M}$ trichostatin for comparison with butyrate. In some experiments, the cells were coincubated with either $5 \mathrm{~g} / \mathrm{mL}$ actinomycin $\mathrm{D}$ (Act $\mathrm{D}$ ) or $8 \mu \mathrm{g} / \mathrm{mL}$ cycloheximide, and butyrate. In other experiments, cells were coincubated with PD98059 $(0-50 \mu \mathrm{M})$, SB203580 $(0-10 \mu \mathrm{M})$, SP600125 $(0-10 \mu \mathrm{M})$, U0126 $(0-50 \mu \mathrm{M})$, or staurosporine $(0-1 \mu \mathrm{M})$, and butyrate.

\section{Assessment of cellular DNA damage}

The structural integrity of DNA in the cells was assessed by the alkaline comet assay, as described previously (Powolny et al., 2001). Briefly, harvested cells were suspended in $0.5 \%$ agarose and aliquots of the suspension pipetted onto frosted glass slides, which had been precoated with the agarose. After congealing of the agarose, the slides were then immersed in ice-cold lysis buffer $(2.5 \mathrm{M} \mathrm{NaCl}, 100 \mathrm{mM}$ NaEDTA, $10 \mathrm{mM}$ Tris, $300 \mathrm{mM} \mathrm{NaOH}, 10 \%$ DMSO, and 1\% Triton $\mathrm{X}-100)$ for $1 \mathrm{~h}$, and then placed in electrophoresis buffer ( $300 \mathrm{mM} \mathrm{NaOH}, 1 \mathrm{mM}$ EDTA) for $20 \mathrm{~min}$ before initiating electrophoresis ( $300 \mathrm{~mA}$ for $20 \mathrm{~min}$ ). After electrophoresis, the slides were submerged twice in neutralization buffer (0.4 M Tris-Cl, pH 7.5) for $5 \mathrm{~min}$, before staining with ethidium bromide and viewing.

\section{Annexin V-FITC binding assay}

HCT-116 colonocytes that had been grown and treated with butyrate in chamber slides were washed twice with cold phosphate-buffered saline (PBS). The washed cells were equilibrated for $10 \mathrm{~min}$ in binding buffer (10 mM HEPES,


tion, the binding buffer was removed from the chamber slide. Then, fresh binding buffer but containing annexin V-Alexa Fluor 488 conjugate (Molecular Probes, Eugene, OR) was pipetted into the chamber slide for $15 \mathrm{~min}$ of incubation. After washing with binding buffer, cells on the slides were viewed on an Olympus BX-60 fluorescence microscope/SPOT digital camera.

\section{TUNEL assay}

HCT-116 colonocytes were washed three times and resuspended in PBS. Aliquots of the cell suspension were pipetted onto lysine-coated glass slides for centrifugation in a Stat Spin Cytofuge. To fix the cells, slides were immersed in $4 \%$ formaldehyde solution in PBS for $25 \mathrm{~min}$ and placed overnight in $70 \%$ ethanol at $-20^{\circ} \mathrm{C}$. The TUNEL (terminal deoxynucleotidyl transferase-mediated dUTP nick end labeling) assay was then performed using a kit along with set of instructions from Promega (Madison, WI). The cell samples were examined by fluorescence microscopy as above.

\section{Determination of GADD153 mRNA expression}

Total RNA was isolated from the cells using an RNeasy Mini kit (Qiagen, Valencia, CA). The expression levels of GADD153 mRNA were determined by multiplex relative RTPCR analysis of total RNA using a Qiagen OneStep RT-PCR kit and gene-specific primers, as described previously (Scott et al., 2005).

\section{Determination of GADD153 protein expression by immunocytofluorescence microscopy and Western blotting analysis}

Cells in LabTek chamber slides were fixed with $4 \%$ formaldehyde in PBS at $25^{\circ} \mathrm{C}$ for $30 \mathrm{~min}$ and then permeabilized with $0.2 \%$ Triton X-100 in PBS at $4^{\circ} \mathrm{C}$ for $5 \mathrm{~min}$. The slides were blocked with $1 \%$ bovine serum albumin (BSA) in PBS at $25^{\circ} \mathrm{C}$ for $3 \mathrm{~h}$. The cells were then incubated overnight at $4{ }^{\circ} \mathrm{C}$ with 1:500 dilution of rabbit polyclonal anti-GADD153 antibody (Santa Cruz Biotechnology, Inc., Santa Cruz, CA) in the blocking buffer. Next, the cells were incubated with 1:1000 dilution of goat anti-rabbit IgG conjugated to Alexa Fluor 488 (Molecular Probes) in the blocking buffer. After thorough washing in PBS, the slides were mounted and cells examined. Western blotting analysis for GADD153 protein was performed as previously described (Scott et al., 2005).

\section{Results}

\section{Evaluation of DNA structural integrity}

As shown in Figure 1A, HCT-116 colonocytes that were exposed to $8 \mathrm{mM}$ butyrate for a range of times $(0-6 \mathrm{~h})$ had nucleoids that were spherical, reflecting no DNA damage. This was also the case with cells exposed to butyrate for $16 \mathrm{~h}$ (data not shown). In contrast and for the purpose of comparison, cells exposed to the reactive oxygen species, hydro- 


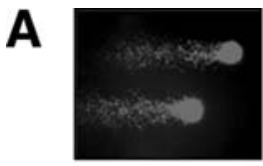

$\mathrm{H}_{2} \mathrm{O}_{2}$

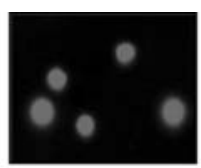

$\mathrm{Oh}$

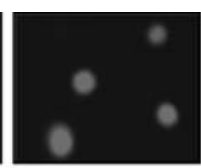

$1 \mathrm{~h}$

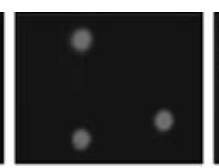

$2 \mathrm{~h}$

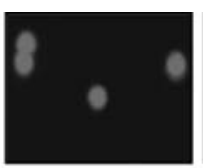

$4 \mathrm{~h}$

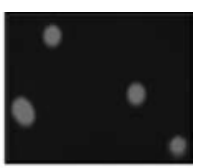

$6 \mathrm{~h}$

incubation time with butyrate

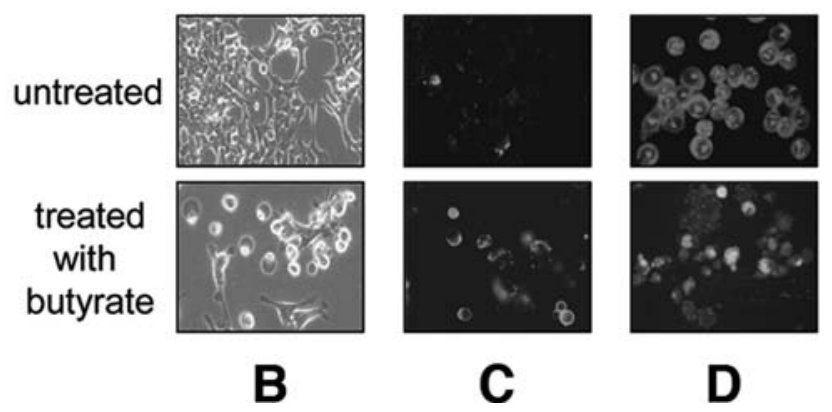

FIG. 1. Absence of detectable DNA damage and display of features characteristic of apoptosis in butyrate-treated HCT-116 colonocytes. In (A), cells were treated with $8 \mathrm{mM}$ butyrate for $0-6 \mathrm{~h}$, and also with $300 \mu \mathrm{M} \mathrm{H}_{2} \mathrm{O}_{2}$ for $0.5 \mathrm{~h}$ as a positive control. Then, the comet assay was performed. In (B), cells were treated with $4 \mathrm{mM}$ butyrate for $36 \mathrm{~h}$ and then examined by light microscopy. In (C), cells were treated with $4 \mathrm{mM}$ butyrate for $24 \mathrm{~h}$, before performing the annexin $\mathrm{V}$ binding assay with subsequent visualization of the cells by fluorescence microscopy using a triple-band filter. In (D), cells were treated with $4 \mathrm{mM}$ butyrate for $48 \mathrm{~h}$, before performing the TUNEL assay with subsequent visualization of the cells by fluorescence microscopy using a triple-band filter. The results are representative of four different experiments.

gen peroxide $\left(\mathrm{H}_{2} \mathrm{O}_{2}\right)$, had nucleoids with the typical comet appearance, reflecting DNA single-strand breaks.

\section{Cellular features characteristic of apoptosis} in HCT-116 colonocytes exposed to butyrate

Some of the HCT-116 colonocytes in the Petri dishes began rounding up within $24 \mathrm{~h}$ of treatment with $4 \mathrm{mM}$ butyrate. When examined later at $36 \mathrm{~h}$ (Fig. 1B), many of the cells showed more distinctive morphological changes-that is, cell shrinkage, cell surface blebbing, and cell break-up into smaller bodies or remnants. It is well known that, during the early stages of apoptosis, phosphatidylserine translocates from the inner to outer leaflet of the plasma membrane. To determine if such a process happens in butyrate-treated HCT-116 colonocytes, the annexin V-Alexa Fluor 488 binding assay was performed to detect any translocated phosphatidylserine at the cell surface. As shown in Figure 1C, more of the butyrate-treated cells than the untreated cells bound the annexin V-Alexa Fluor 488 probe (giving them a bright green fluorescence at the cell surface).

It is also widely known that DNA becomes fragmented during the late stages of apoptosis. To determine if DNA fragmentation occurs in butyrate-treated HCT-116 colonocytes, the TUNEL assay was performed. As shown in Figure $1 \mathrm{D}$, more of the butyrate-treated cells than the untreated cells tested positive, as indicated specifically by those cells emitting bright green fluorescence (against the red background fluorescence produced by counterstaining all the cells with propidium iodide).

\section{Effect of butyrate on GADD153 mRNA and protein expression in HCT-116 colonocytes}

Upon performing multiplex relative RT-PCR analysis using gene-specific primers for GADD153 and the internal control $\beta$-actin, it was found that GADD153 mRNA was expressed constitutively in HCT-116 colonocytes (Fig. 2A, B). However, exposing the cells to butyrate increased GADD153 mRNA expression. This effect of butyrate was both concentration dependent (Fig. 2A) and time dependent (Fig. 2B).

To determine if butyrate caused any changes in GADD153 protein expression in HCT-116 colonocytes, immunocytofluorescence microscopy was performed using an anti-GADD153 polyclonal antibody and the counterstain, propidium iodide. Representative results are shown in Figure $2 \mathrm{C}$. No visual evidence of GADD153 protein expression can be seen in any of the cells of the untreated or control sample (left image), as indicated by the absence of green fluorescence. In the butyratetreated sample (right image), some of the cells expressed GADD153 protein to varying extents, as indicated by the presence of green fluorescence. Expression of GADD153 protein was also assessed by western blotting analysis (Fig. 2D), which revealed that induction of GADD153 protein by butyrate was only slight (in comparison to cells exposed to DOC as a positive control) and not clearly concentration-dependent. It is possible that a large portion of the GADD153 transcript is not being translated. Alternatively, there could be rapid proteosomal turnover of part of the pool of newly synthesized GADD153 protein.

\section{Effect of actinomycin $D$ and cycloheximide on GADD153 mRNA upregulation caused by butyrate in HCT-116 colonocytes}

To determine whether butyrate increases GADD153 mRNA at the level of transcription, an experiment using the RNA synthesis inhibitor, actinomycin D, was performed (Fig. 3A). As before, butyrate increased GADD153 mRNA expression in HCT-116 colonocytes (lane 2) when compared to control cells (lane 1). However, actinomycin D prevented 

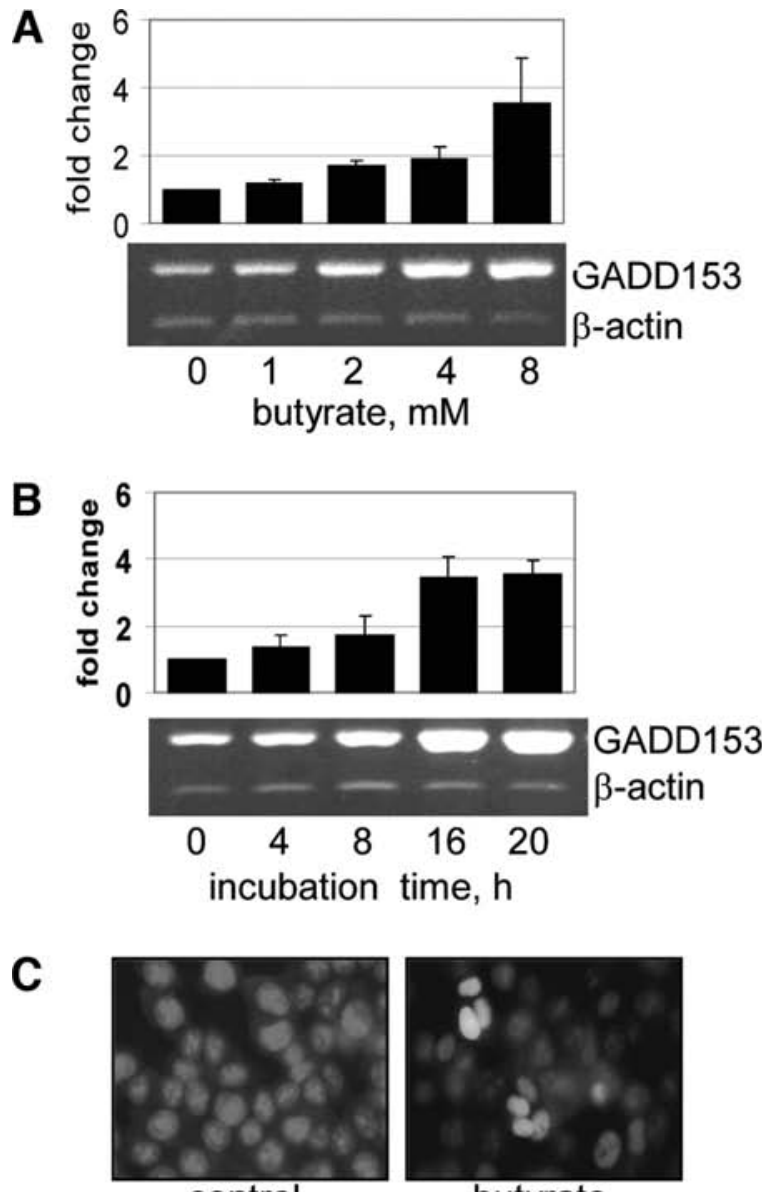

control

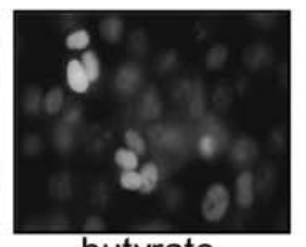

butyrate

D

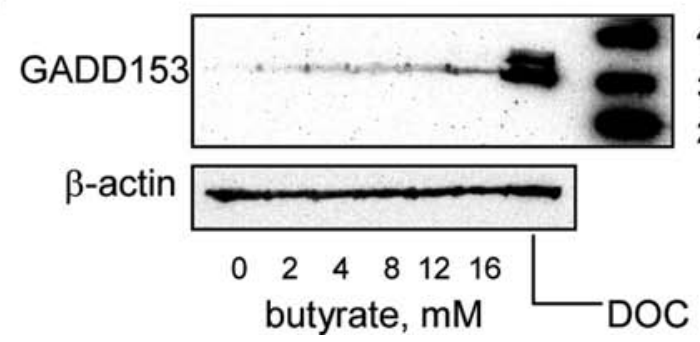

FIG. 2. Increased expression of GADD153 mRNA and protein in butyrate-treated HCT-116 colonocytes. Cells were treated with $0-8 \mathrm{mM}$ butyrate for $14 \mathrm{~h}$ (A) or with $4 \mathrm{mM}$ butyrate for $0-20 \mathrm{~h}(\mathbf{B})$. Then, multiplex relative RT-PCR analysis was performed to determine expression of the target gene, GADD153, and internal control gene, $\beta$-actin. The bar graphs above each of the representative gel images denote the changes (fold differences, average \pm SD) in GADD153 expression (normalized against $\beta$-actin) relative to control. In (C), cells were treated with $4 \mathrm{mM}$ butyrate for $15 \mathrm{~h}$. Then, immunocytofluorescence microscopy was performed to analyze GADD153 protein expression, using anti-GADD153 primary antibody along with a secondary antibody conjugated to Alexa Fluor 488 (green fluorescence). Counterstaining was achieved with propidium iodide (red fluorescence). The cells were viewed with a triple-band filter. In (D), cells were treated with $0-16 \mathrm{mM}$ butyrate for $20 \mathrm{~h}$ and western blotting analysis performed, incorporating cells exposed for $4 \mathrm{~h}$ to $300 \mu \mathrm{M}$ deoxycholate (DOC) as a positive control. All results are representative of four different experiments.

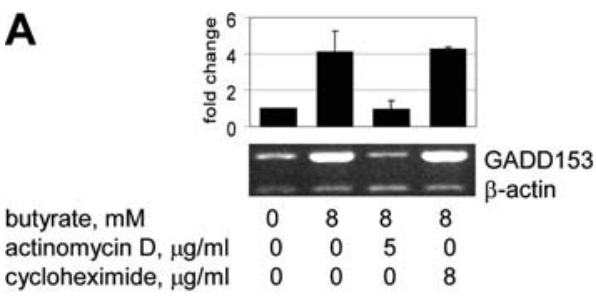

B

butyrate, $\mathrm{mM}$

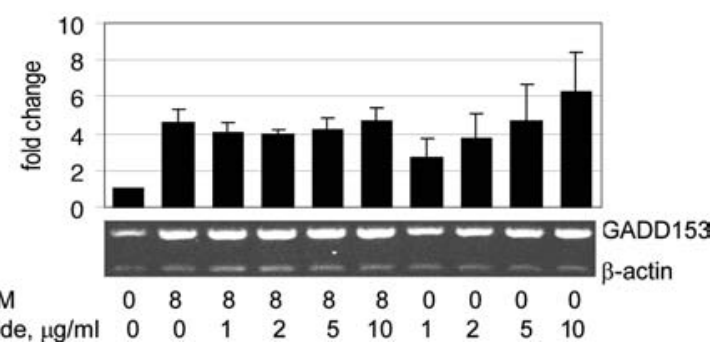

FIG. 3. Impact of actinomycin $\mathrm{D}$ and cycloheximide on butyrate-induced GADD153 mRNA upregulation in HCT-116 colonocytes. The cells were treated with butyrate for $8 \mathrm{~h}$, with either actinomycin $\mathrm{D}$ or cycloheximide being added at the 4-h mid-time point. Then, multiplex relative RT-PCR analysis was performed to determine expression of the target gene, GADD153, and internal control gene, $\beta$-actin (A). In (B), a similar experiment was performed but using only cycloheximide at varying concentrations. The bar graphs above each of the representative gel images denote the changes (fold differences, average $\pm \mathrm{SD}$ ) in GADD153 expression (normalized against $\beta$-actin) relative to control. The results are representative of three different experiments.

the GADD153 mRNA upregulation caused by butyrate (lane 3). Therefore, these data would imply that butyrate-induced GADD153 mRNA upregulation is due more likely to increased synthesis, rather than to greater stability, of the transcript.

To determine if protein synthesis is required for butyrateinduced GADD153 mRNA upregulation, the standard protein synthesis inhibitor, cycloheximide, was considered as being the ideal tool to use. The results of the experiment turned out to be ambiguous, but nevertheless yielded some additional information regarding the identity of other substances capable of modulating GADD153 mRNA expression. In this experiment, we opted to incubate HCT-116 colonocytes with butyrate at $8 \mathrm{mM}$, which is the highest concentration tested in the present study. As shown in Figure 3A, it would initially appear that cycloheximide $(8 \mu \mathrm{g} / \mathrm{mL})$ did not affect the capacity of butyrate to increase GADD153 mRNA expression, in comparing lane 4 with lane 2 . As can be seen in Figure 3B, a range of cycloheximide concentrations $(0-10 \mu \mathrm{g} / \mathrm{mL})$ was also tested, and cycloheximide had no observable effects on butyrateinduced upregulation of GADD153 mRNA expression, in comparing lane 2 with lanes 3-6. However, further examination of Figure $3 \mathrm{~B}$ reveals that cycloheximide by itself actually increased GADD153 mRNA expression noticeably, in comparing lane 1 with lanes 7-10. Therefore, because of this interfering, independent upregulatory effect of cycloheximide on GADD153 mRNA expression, no clear conclusion can be drawn concerning a requirement for protein synthesis in butyrate-induced GADD153 mRNA upregulation. 
A

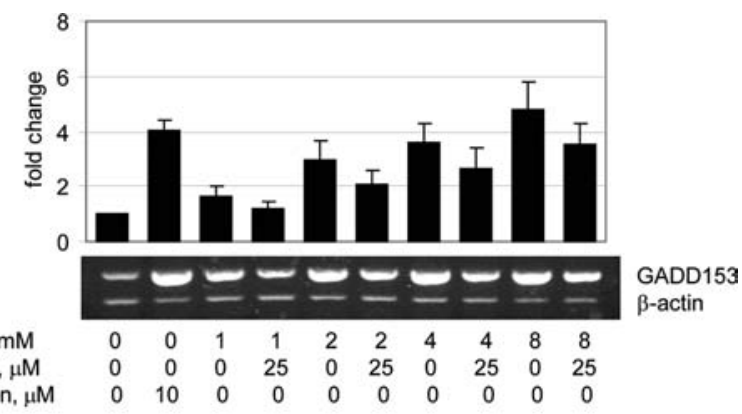

PD98059, $\mu \mathrm{M}$

trichostatin, $\mu \mathrm{M}$

B

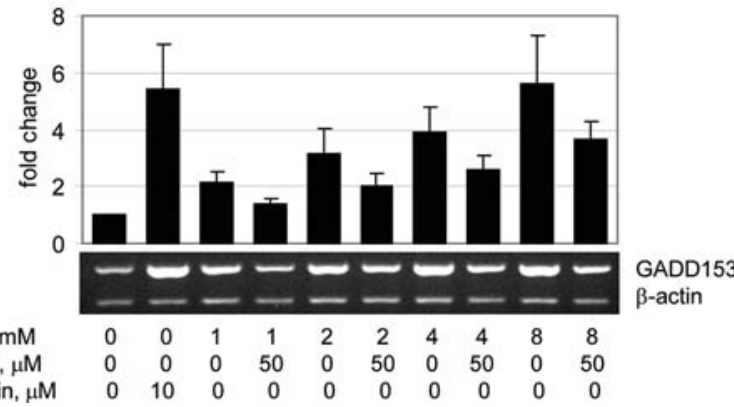

FIG. 4. Effect of PD98059 on butyrate-induced upregulation of GADD153 mRNA expression in HCT-116 colonocytes. HCT-116 colonocytes were treated with $0-8 \mathrm{mM}$ butyrate for $8 \mathrm{~h}$, with trichostatin serving as a comparative positive control, in the absence and presence of $25 \mu \mathrm{M}$ PD98059 (A) or $50 \mu \mathrm{M}$ PD98059 (B). Then, multiplex relative RT-PCR analysis was performed to determine expression of the target gene, GADD153, and internal control gene, $\beta$-actin. The bar graphs above each of the representative gel images denote the changes (fold differences, average \pm SD) in GADD153 expression (normalized against $\beta$-actin) relative to control. The results are representative of three different experiments.

\section{Effect of protein kinase inhibitors on butyrate-induced upregulation of GADD153 mRNA and protein expression in HCT-116 colonocytes}

MAPKs have been reported to be involved in upregulating GADD gene expression (Rolli-Derkinderen and Gaestel, 2000; Oh-Hashi et al., 2001; Sarkar et al., 2002). Hence, we first asked the question if a selective chemical inhibitor of ERK, specifically PD98059, might have an inhibitory effect on butyrate-induced GADD153 mRNA upregulation. At a concentration of $25 \mu \mathrm{M}, \mathrm{PD} 98059$ noticeably blunted GADD153 mRNA upregulation caused by $1,2,4$, or $8 \mathrm{mM}$ butyrate (Fig. 4A). Further, testing PD98059 at double the concentration $(50 \mu \mathrm{M})$ did not produce an additional blunting effect of significant proportion on butyrate-induced GADD153 mRNA upregulation (Fig. 4B). Because butyrate is often used as an inhibitor of histone deacetylase (Nakano et al., 1997; Archer et al., 1998), this prompted accompanying experiments (Fig. 4A, B) to show that trichostatin, also a known histone deacetylase inhibitor, increases GADD153 mRNA expression as well, although further investigation of trichostatin's effect was not pursued.

In performing an experiment to determine the impact of PD98059 on butyrate-induced upregulation of GADD153 protein expression (Fig. 5A), immunocytofluorescence microscopy again revealed that cells in the control sample expressed very little, if any, GADD153 protein, as indicated by

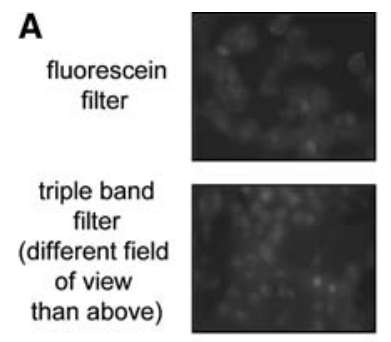

control
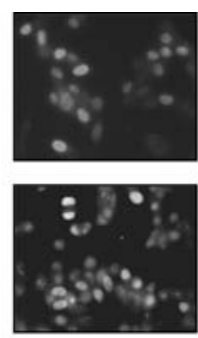

butyrate $(4 \mathrm{mM})$
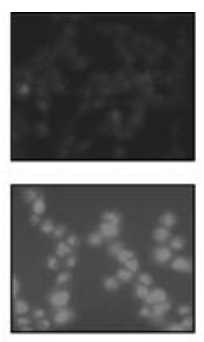

butyrate (4 mM) PD98059 $(25 \mu \mathrm{M})$
B

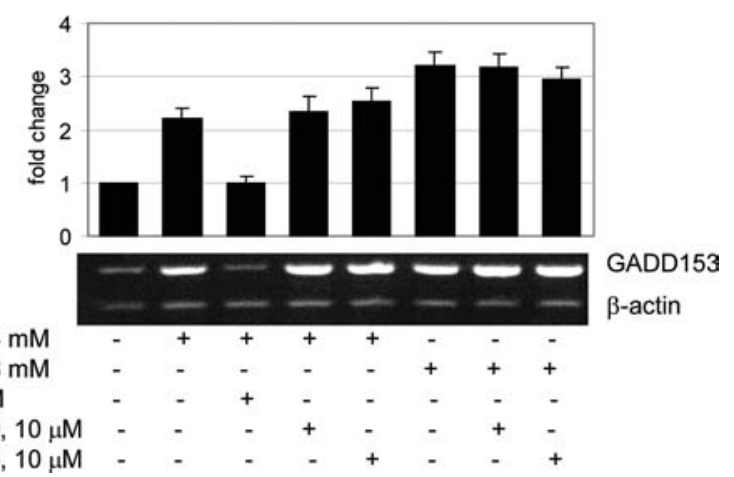

FIG. 5. Effects of PD98059, SB203580, SP600125, and staurosporine (SRS) on butyrate-induced upregulation of GADD153 protein or mRNA expression in HCT-116 colonocytes. In panel (A), HCT-116 colonocytes were treated with $4 \mathrm{mM}$ butyrate for $14 \mathrm{~h}$ in the absence and presence of $25 \mu \mathrm{M}$ PD98059. Then, immunocytofluorescence microscopy was performed to assess GADD153 protein expression, using anti-GADD153 primary antibody along with a secondary antibody conjugated to Alexa Fluor 488 (green fluorescence). Counterstaining was achieved with propidium iodide (red fluorescence). The cells were viewed with a fluoroscein filter and also with a triple-band filter. In panel (B), HCT-116 colonocytes were incubated with butyrate for $8 \mathrm{~h}$, but with staurosporine (SRS), SB203580, or SP600125 being added at the 4-h mid-time point to lessen potential cytotoxicity. Then, multiplex relative RT-PCR analysis was performed to determine expression of the target gene, GADD153, and internal control gene, $\beta$-actin. The bar graph above the representative gel image denotes the changes (fold differences, average \pm $\mathrm{SD})$ in GADD153 expression (normalized against $\beta$-actin) relative to control. The results are representative of three different experiments.

the presence of only dim green background fluorescence, when viewed with the fluoroscein filter (left upper image). In the butyrate-treated sample, some of the cells exhibited bright green fluorescence and hence expressed GADD153 protein (middle upper image). Most importantly, it can be seen that PD98059 was effective in essentially preventing the induction of GADD153 protein expression caused by butyrate, as indicated by the absence of bright green fluorescence (right upper image).

To help confirm the microscopic findings above, other slides were prepared, doubly stained, and then examined (Fig. 5A, lower set of images) using a triple-band filter to permit simultaneous observation of red fluorescence (i.e., cells counterstained with propidium iodide to reveal their presence and to verify that a similar number of cells was 
A

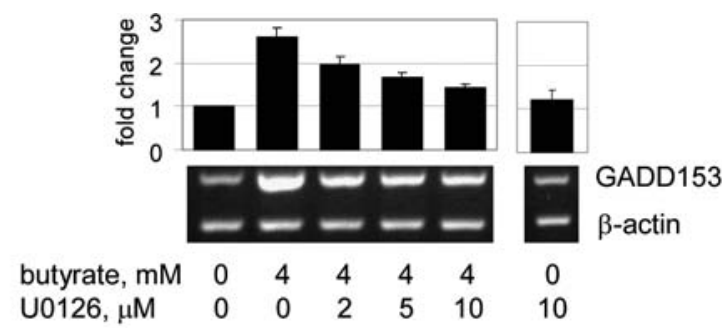

B



control
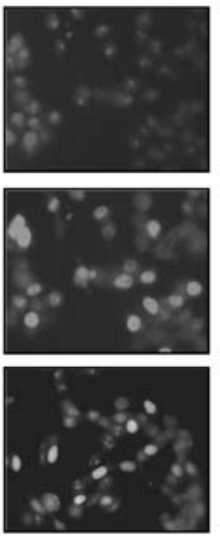

butyrate
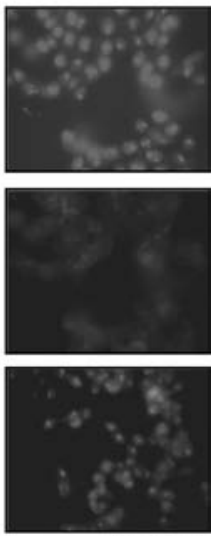

butyrate/U0126

FIG. 6. Selective MEK inhibitor, U0126, blunts induction of GADD153 mRNA and protein caused by butyrate in HCT-116 colonocytes. The cells were treated with butyrate for $15 \mathrm{~h}$ in the absence and presence of U0126. Then, multiplex relative RTPCR analysis was performed to determine GADD153 mRNA expression (A). The bar graph above the representative gel image denotes the changes (fold differences, average \pm SD) in GADD153 expression (normalized against $\beta$-actin) relative to control. Also, cells were treated with butyrate for $14 \mathrm{~h}$ in the absence and presence of U0126. Then, immunocytofluorescence microscopy was performed to analyze GADD153 protein expression (B), using anti-GADD153 primary antibody along with a secondary antibody conjugated to Alexa Fluor 488 (green fluorescence). Counterstaining was achieved with propidium iodide (red fluorescence). The cells were viewed with three different filters as indicated above for comparison. The results are representative of three different experiments.

present in the fields of view) and any green fluorescence (i.e., immunoreactivity of the cells with anti-GADD153 antibody/anti-rabbit IgG conjugated to Alexa Fluor 488, indicative of the presence of GADD153 protein). In agreement with the results shown in the upper images of Figure 5A, butyrate induced GADD153 protein expression to some extent (middle lower image). This effect of butyrate was prevented by PD98059 (right lower image).

Because the ERK inhibitor, PD98059, produced positive results, selective chemical inhibitors of other MAPK were evaluated similarly to gain more insight on the potential importance of MAPK in mediating the effect of butyrate on GADD153 mRNA expression. At the concentrations tested, neither SB203580 (p38 MAPK inhibitor) nor SP600125 (JNK inhibitor) blunted butyrate-induced upregulation of GADD153 mRNA upregulation (Fig. 5B), in contrast to the blunting effect previously found with PD98059 (Fig. 4A, B). In fact, SB203580 and SP600125 were subsequently found to increase GADD153 mRNA expression, when each inhibitor was tested alone (data

not shown). To evaluate a potential involvement of PKC in mediating the effect of butyrate on GADD153 mRNA expression, an experiment with staurosporine (general or broadspectrum PKC inhibitor) was simultaneously conducted. Staurosporine (SRS) prevented the increase in GADD153 mRNA expression caused by butyrate (Fig. 5B). However, it was also subsequently found that staurosporine by itself $(1 \mu \mathrm{M})$ seemed to produce morphological signs of apoptosis in HCT116 colonocytes (data not shown), which is consistent with the reported effects of staurosporine on HT-29 human colon adenocarcinoma cells (Qiao et al., 1996).

Finally, to determine if MEK, which is immediately upstream of ERK in the MAPK molecular cascade, might be important in mediating upregulation of GADD153 mRNA expression by butyrate, similar experiments as above were performed utilizing the MEK inhibitor, U0126. As shown in Figure 6A, U0126 blunted butyrate-induced upregulation of GADD153 mRNA expression, and by itself did not affect GADD153 mRNA. Further, as can be seen by the immunocytofluorescence microscopy images obtained with three different filters (Fig. 6B), the overall interpretation is that U0126 prevented the perceptible upregulation of GADD153 protein expression caused by butyrate. The rhodamine filter-based images show the cells counterstained with propidium iodide to reveal similar cell densities among the three samples analyzed. Using a fluoroscein filter to examine the exact same field of view, any GADD153 protein being expressed by the cells can be visualized, as is the case with those cells emitting the bright green fluorescence. Examining different fields of view for the three samples with a triple-band filter corroborated the observations above.

\section{Discussion}

Butyrate is often investigated because of a particular interest in its possible chemopreventive effects against colon cancer. Eating foods rich in dietary fiber supposedly lowers the risk of colon cancer (Bingham et al., 2003). One plausible explanation is that colonic microbes degrade the dietary fiber to produce a beneficial metabolite, specifically butyrate, which then suppresses the growth or proliferation of progenitor colon cancer cells that otherwise would replicate to eventually form a tumor. This notion is based partly on several studies (Nakano et al., 1997; Archer et al., 1998; Litvak et al., 1998; Ruemmele et al., 2003), where butyrate was found to inhibit cultured colon cancer cells from proliferating.

In the present study, HCT-116 human colon adenocarcinoma cells exposed to millimolar concentrations of butyrate within the physiological range (Topping and Clifton, 2001) were studied because of the obvious relevance to the topic of dietary fiber-derived short-chain fatty acids and colon cancer (Bingham et al., 2003). Evidence of apoptosis was found in HCT-116 colonocytes exposed to butyrate. More specifically, examination of the cells by light microscopy and also the results of the annexin $\mathrm{V}$ binding and TUNEL assays revealed signs of apoptosis. These current findings corroborate and expand similar findings by other workers studying the apoptotic effects of butyrate (Ruemmele et al., 2003). It is well known that oxidative damage to DNA can lead to apoptosis. Hence, in causing apoptosis, butyrate could have created a state of oxidative stress resulting in DNA damage, since it has been reported that butyrate can induce the pro- 
duction of reactive oxygen species (Jeng et al., 2006). However, no DNA damage was detected in HCT-116 colonocytes exposed to butyrate, which is consistent with a study by other workers (Rosignoli et al., 2001) using HT29 colonocytes exposed to butyrate. Nevertheless, butyrate increased the expression of GADD153 mRNA, although GADD153 protein was only slightly increased. Therefore, the upregulation of GADD153 gene expression caused by butyrate is apparently not a molecular response of the cells to DNA damage, which was absent in butyrate-treated cells. One well-known function of GADD153 is that it acts as a dominant-negative inhibitor of the C/EBP family of transcription factors (Ron and Habener, 1992), thereby enabling GADD153 protein to indirectly regulate $\mathrm{C} / \mathrm{EBP}$-responsive genes. Other studies (Conn et al., 2002; Kim et al., 2002; Lengwehasatit and Dickson, 2002; Xia et al., 2002) have suggested that GADD153 somehow mediates the critical early events leading to the initiation of apoptosis. However, in view of the current finding that GADD153 protein was only slightly increased in HCT-116 cells exposed to butyrate, it cannot be concluded that GADD153 plays a pivotal role in butyrateinduced apoptosis.

The ability of actinomycin D to essentially prevent butyrate-induced upregulation of GADD153 mRNA expression supports the probability that the effect of butyrate on GADD153 mRNA was due primarily to increased transcription rather than greater mRNA stability. However, it is known that GADD153 mRNA levels can be upregulated in distinct ways depending on the experimental conditions. For example, greater GADD153 mRNA expression in leucine-deprived cells was due to both increased transcription and mRNA stability (Bruhat et al., 1997), whereas glutamine deprivation increased GADD153 mRNA expression principally by stabilizing mRNA transcript (Abcouwer et al., 1999). In contrast, iron-deficient cells had greater GADD153 mRNA expression, which was concluded to be attributed mainly to increased transcription (Pan et al., 2004).

Regarding possible involvement of upstream protein kinases in mediating butyrate-induced GADD153 mRNA upregulation, MAPKs were given prime consideration for current investigation because of other studies that have identified a role for MAPK in upregulating GADD153 gene expression as caused by other agents. As a case in point, anisomycin caused activation of p38 MAPK in Jurkat T-lymphocytes while also increasing GADD153 mRNA expression (Rolli-Derkinderen and Gaestel, 2000). However, the effect of anisomycin on GADD153 mRNA was prevented when the cells were pretreated with a selective chemical inhibitor of p38 MAPK. Similarly, chemical inhibition of p38 MAPK suppressed GADD153 mRNA upregulation caused by peroxynitrite in human neuroblastoma SH-SY5Y cells (Oh-Hashi et al., 2001). In another study, but with human melanoma cells (Sarkar et al., 2002), ectopic overexpression of the interleukin-24 (IL-24) gene via an adenovirus carrying the IL-24 gene resulted in GADD153 gene upregulation that was also found to be dependent on p38 MAPK. Recently, it was reported (Li and Holbrook, 2004) that exposing primary rat hepatocytes to agents that induce stress to the endoplasmic reticulum results in elevated GADD153 expression, which was concluded to prominently involve JNK.

Taken together, the above studies (Rolli-Derkinderen and Gaestel, 2000; Oh-Hashi et al., 2001; Sarkar et al., 2002;
Li and Holbrook, 2004) would generate the thought that MAPK could be involved in butyrate-induced upregulation of GADD153 gene expression as well. Based on the current results of the experiments utilizing selective chemical inhibitors of p38 MAPK and JNK, it seems that p38 MAPK and JNK do not play a role. Previously, butyrate was reported to cause activation of ERK in K562 human erythroleukemic cells (Rivero and Adunyah, 1996). Thus, in evaluating the effects of other selective chemical inhibitors of MAPK, it was found that the ERK inhibitor, PD98059, but also the MEK inhibitor, U0126, noticeably attenuated the increased GADD153 mRNA and protein expression caused by butyrate. Therefore, these two new findings suggest that butyrateinduced upregulation of GADD153 mRNA expression involved at least the MEK/ERK branch of the MAPK cascade, which adds to the base of knowledge on the signal transduction events enabling butyrate to influence the GADD153 gene. In light of the rather diverse involvement of p38 MAPK (Rolli-Derkinderen and Gaestel, 2000; Oh-Hashi et al., 2001; Sarkar et al., 2002), JNK (Li and Holbrook, 2004), and ERK as currently reported herein in mediating GADD153 gene upregulation, it appears that the particular experimental conditions will largely determine which of the MAPK will be pivotal in mediating the induction of GADD153 gene expression. In any event, conventional wisdom holds that activated MAPK would eventually promote activation of specific transcription factors leading to transcriptional activation of the GADD153 gene.

On the other hand, it is conceivable that other protein kinases are important in mediating the upregulatory effects of butyrate on GADD153 gene expression. In seeming support of this possibility with respect to PKC, it was observed that the general PKC inhibitor, staurosporine, was able to prevent butyrate-induced GADD153 mRNA upregulation in HCT-116 colonocytes. However, this observation should be interpreted with caution because the results were achieved with only relatively high concentrations of staurosporine, which can also induce cell cycle arrest and apoptosis (Qiao et al., 1996). As such, the role of PKC in mediating butyrate-induced GADD 153 gene activation remains uncertain and will require further investigation, especially in view of the numerous known isoforms of PKC. Nevertheless, the present study is significant because it sheds light on some aspects of a signaling cascade facilitating GADD153 upregulation as caused by butyrate.

\section{Acknowledgments}

The project was supported by the National Research Initiative of the USDA Cooperative State Research, Education and Extension Service (grant number 2006-35200-16578), and also by the North Carolina Agricultural Research Service (NC06659).

\section{Author Disclosure Statement}

No competing financial interests exist.

\section{References}

Abcouwer, S.F., Schwarz, C., and Meguid, R.A. (1999). Glutamine deprivation induces the expression of GADD45 and GADD153 primarily by mRNA stabilization. J Biol Chem 274, 28645-28651. 
Archer, S.Y., Meng, S., Shei, A., and Hodin, R.A. (1998). p21 (WAF1) is required for butyrate-mediated growth inhibition of human colon cancer cells. Proc Natl Acad Sci USA 95, 6791-6796.

Bai, L., and Merchant, J.L. (2000). Transcription factor ZBP-89 cooperates with histone acetyltransferase p300 during butyrate activation of p21waf1 transcription in human cells. J Biol Chem 275, 30725-30733.

Bingham, S.A., Day, N.E., Luben, R., Ferrari, P., Slimani, N., Norat, T., Clavel-Chapelon, F., Kesse, E., Nieters, A., Boeing, H., Tjonneland, A., Overvad, K., Martinez, C., Dorronsoro, M., Gonzalez, C.A., Key, T.J., Trichopoulou, A., Naska, A., Vineis, P., Tumino, R., Krogh, V., Bueno-de-Mesquita, H.B., Peeters, P.H., Berglund, G., Hallmans, G., Lund, E., Skeie, G., Kaaks R., and Riboli, E. (2003). European Prospective Investigation into Cancer and Nutrition. Dietary fibre in food and protection against colorectal cancer in the European Prospective Investigation into Cancer and Nutrition (EPIC): an observational study. Lancet 361, 1496-1501.

Bruhat, A., Jousse, C., Wang, X.Z., Ron, D., Ferrara, M., and Fafournoux, P. (1997). Amino acid limitation induces expression of CHOP, a CCAAT/enhancer binding protein-related gene, at both transcriptional and post-transcriptional levels. J Biol Chem 272, 17588-17593.

Conn, K.J., Gao, W.W., Ullman, M.D., McKeon-O'Malley, C., Eisenhauer, P.B., Fine, R.E., and Wells, J.M. (2002). Specific up-regulation of GADD153/CHOP in 1-methyl-4-phenylpyridinium-treated SH-SY5Y cells. J Neurosci Res 68, 755-760.

Daly, K, Shirazi-Beechey, S.P. (2006). Microarray analysis of butyrate regulated genes in colonic epithelial cells. DNA Cell Biol 25, 49-62.

Jeng, J.H., Kuo, M.Y., Lee, P.H., Wang, Y.J., Lee, M.Y., Lee, J.J., Lin, B.R., Tai, T.F., and Chang, M.C. (2006). Toxic and metabolic effect of sodium butyrate on SAS tongue cancer cells: role of cell cycle deregulation and redox changes. Toxicology 223, 235-247.

Kim, D.-G., You, K.-R., Liu, M.-J., Choi, Y.-K., and Won, Y.-S. (2002). GADD153-mediated anticancer effects of N-(4hydroxyphenyl)retinamide on human hepatoma cells. J Biol Chem 277, 38930-38938.

Lengwehasatit, I., and Dickson, A.J. (2002). Analysis of the role of GADD153 in the control of apoptosis in NS0 myeloma cells. Biotechnol Bioeng 80, 719-730.

Li, J., and Holbrook, N.J. (2004). Elevated gadd153/chop expression and enhanced c-Jun N-terminal protein kinase activation sensitizes aged cells to ER stress. Exp Gerontol 39, 735-744.

Litvak, D.A., Evers, B.M., Hwang, K.O., Hellmich, M.R., Ko, T.C., and Townsend, Jr., C.M. (1998). Butyrate-induced differentiation of Caco-2 cells is associated with apoptosis and early induction of p21Waf1/Cip1 and p27Kip1. Surgery 124, 161-169.

Nakano, K., Mizuno, T., Sowa, Y., Orita, T., Yoshino, T., Okuyama, Y., Fujita, T., Ohtani-Fujita, N., Matsukawa, Y., Tokino, T., Yamagishi, H., Oka, T., Nomura, H., and Sakai, T. (1997). Butyrate activates the WAF1/Cip1 gene promoter through Sp1 sites in a p53-negative human colon cancer cell line. J Biol Chem 272, 22199-22206.

Oh-Hashi, K., Maruyama, W., and Isobe, K. (2001). Peroxynitrite induces GADD34, 45, 153 via p38 MAPK in human neuroblastoma SH-SY5Y cells. Free Radic Biol Med 30, 213-221.

Pan, Y.J., Hopkins, R.G., and Loo, G. (2004). Increased GADD153 gene expression during iron chelation-induced apoptosis in Jurkat T-lymphocytes. Biochim Biophys Acta 1691, 41-50.

Powolny, A., Xu, J., and Loo, G. (2001). Deoxycholate induces DNA damage and apoptosis in human colon epithelial cells expressing either mutant or wild-type p53. Int J Biochem Cell Biol 33, 193-203.

Qiao, L., Koutsos, M., Tsai, L.L., Kozoni, V., Guzman, J., Shiff, S.J., and Rigas, B. (1996). Staurosporine inhibits the proliferation, alters the cell cycle distribution and induces apoptosis in HT-29 human colon adenocarcinoma cells. Cancer Lett 107, 83-89.

Rivero, J.A., and Adunyah, S.E. (1996). Sodium butyrate induces tyrosine phosphorylation and activation of MAP kinase (ERK-1) in human K562 cells. Biochem Biophys Res Commun 224, 796-801.

Rolli-Derkinderen, M., and Gaestel, M. (2000). p38/SAPK2dependent gene expression in Jurkat T cells. Biol Chem 381, 193-198.

Ron, D., and Habener, J.F. (1992). CHOP, a novel developmentally regulated nuclear protein that dimerizes with transcription factors $\mathrm{C} / \mathrm{EBP}$ and LAP and functions as a dominant-negative inhibitor of gene transcription. Genes Dev 6, 439-453.

Rosignoli, P., Fabiani, R., de Bartolomeo, A., Spinozzi, F., Agea, E., Pelli, M.A., and Morozzi, G. (2001). Protective activity of butyrate on hydrogen peroxide-induced DNA damage in isolated human colonocytes and HT29 tumour cells. Carcinogenesis 22, 1675-1680.

Ruemmele, F.M., Schwartz, S., Seidman, E.G., Dionne, S., Levy, E., and Lentze, M.J. (2003). Butyrate induced Caco-2 cell apoptosis is mediated via the mitochondrial pathway. Gut 52, 94-100.

Sarkar, D., Su, Z.Z., Lebedeva, I.V., Sauane, M., Gopalkrishnan, R.V., Valerie, K., Dent, P., and Fisher, P.B. (2002). mda-7 (IL-24) mediates selective apoptosis in human melanoma cells by inducing the coordinated overexpression of the GADD family of genes by means of p38 MAPK. Proc Natl Acad Sci USA 99, 10054-10059.

Scott, D.W., Mutamba, S., Hopkins, R.G., and Loo, G. (2005). Increased GADD gene expression in human colon epithelial cells exposed to deoxycholate. J Cell Physiol 202, 295-303.

Sowa, Y., Orita, T., Minamikawa, S., Nakano, K., Mizuno, T., Nomura, H., and Sakai, T. (1997). Histone deacetylase inhibitor activates the WAF1/Cip1 gene promoter through the Sp1 sites. Biochem Biophys Res Commun 241, 142-150.

Sowa, Y., Orita, T., Minamikawa-Hiranabe, S., Mizuno, T., Nomura, H., and Sakai, T. (1999). Sp3, but not Sp1, mediates the transcriptional activation of the $\mathrm{p} 21 / \mathrm{WAF} 1 / \mathrm{Cip} 1$ gene promoter by histone deacetylase inhibitor. Cancer Res 59, 42664270.

Topping, D.L., and Clifton, P.M. (2001). Short-chain fatty acids and human colonic function: roles of resistant starch and nonstarch polysaccharides. Physiol Rev 81, 1031-1064.

Xia, Y., Wong, N.S., Fong, W.F., and Tideman, H. (2002). Upregulation of GADD153 expression in the apoptotic signaling of N-(4-hydroxyphenyl)retinamide (4HPR). Int J Cancer 102, 7-14.

Address reprint requests to: George Loo, Ph.D. Cellular and Molecular Nutrition Research Laboratory Department of Nutrition

University of North Carolina at Greensboro Greensboro, NC 27402-6170

E-mail: g_loo@uncg.edu

Received for publication June 6, 2008; received in revised form July 3, 2008; accepted July 3, 2008. 
Copyright of DNA \& Cell Biology is the property of Mary Ann Liebert, Inc. and its content may not be copied or emailed to multiple sites or posted to a listserv without the copyright holder's express written permission. However, users may print, download, or email articles for individual use. 